and for the present strain rate field becomes

$$
\dot{D}_{i}=\frac{e V \sigma_{0} h}{\left(e+S_{2}\right)^{2}}\left(2 e+S_{2}\right)\left(A-C_{0}-S_{1}\right)
$$

The external work rate, $\dot{D}_{e}$, includes $(a)$ the total potential energy-loss rate, and $(b)$ the rate of conversion of potential energy into kinetic energy. Evidently, $\dot{D}_{e}$, is given by the difference of $(a)$ and (b), or

$$
\dot{D}_{e}=2\left(A-C_{0}\right) h \sigma_{0} V \frac{g-\dot{V}}{g}
$$

In establishing the energy criterion for crack propagation, three more energy terms are considered. It is shown that these terms are quite insignificant compared to internal energy dissipation rate and external work rate and may be neglected. The origin of these additional energy terms may be traced to

(1) Kinetic energy due to displacements in front of the propagating crack,

(2) Elastic strain energy stored in the specimen and its availability for crack extension, and

(3) Energy absorption due to lateral buckling of the specimen during the process of crack propagation.

The energy contribution due to (1) above was calculated by Anderson ${ }^{8}$ for an ideally elastic and brittle material. The expression for kinetic-energy rate resulting from displacements around the crack was found to be

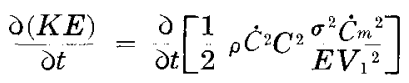

However, as Orowan ${ }^{2}$ pointed out, ductile fracture is a process whose velocity is determined by applied strain rate, and for the present dead-weight loading apparatus, the velocity $V$ is small. Accordingly $\dot{C}$ is also small, and it is possible to show that eq $(A-8)$ is of the order $10^{-3}$ of eq $(A-6)$ from dimensional analysis.

In connection with energy source (2) Griffith ${ }^{1}$ showed that the elastic energy available to drive cracks in brittle materials is approximately equal to $0.3 \pi\left(\sigma^{2} C^{2} h / E\right)$. From this expression the release rate of elastic energy becomes $0.6 \pi \sigma^{2} C \dot{C} h / E$ which, again, can be neglected by comparison.

The energy rate associated with lateral buckling has been previously discussed and is negligible when compared to internal-dissipation and external-work rates.

Neglecting the energy terms (1), (2) and (3), the energy criteria for crack propagation may be expressed as follows:

Internal-energy dissipation rate $\left(\dot{D_{i}}\right)=$ external-work rate $\left(\dot{D_{e}}\right)$.

Substituting the values for $\dot{D}_{i}$ and $\dot{D}_{e}$ from eq $(A-6)$ and $(A-7)$, respectively, in the energy criteria, the following expression for $S_{1}$ may be obtained:

$$
S_{1}=\frac{\left(e+S_{2}\right)^{2}\left(A-C_{0}\right)}{e\left(2 e+S_{2}\right)}\left[\frac{e\left(2 e+S_{2}\right)}{\left(e+S_{2}\right)_{2}}-\frac{2(g-\dot{V})}{g}\right]
$$

Equation $(A-9)$ gives $S_{1}$ as a function of $S_{2}, \dot{V}$ and $e$. The crack-propagation velocity is now obtained by simply differentiating eq $(A-9)$ with respect to time.

In the preceding analysis, the existence of the plastic-flow region has been tacitly assumed. No attempt was made or shall be made in what follows to find an empirical relationship for the plastic-flow region in terms of known or easily determined material variables. However, a few factors that might influence its extent may be noted here in passing. These factors include test temperature, specimen dimensions (particularly thickness), initial crack length, yield point and ultimate strength of the material. The question whether an empirical relationship for the determination of $e$ involves only the aforementioned material variables and test conditions can only be answered by a carefully planned experimental program. Upon a little reflection, it would seem reasonable that tests on specimens made out of different metals, dimensions, initial crack lengths and test temperatures should yield the maximum amount of information.

\title{
ERRATA:
}

\section{Anticlastic Behavior of Flat Plates}

by D. G. Bellow, G. Ford and J. S. Kennedy

We regret that an error appeared in Fig. 1 on page 228 of the July issue of EXPERIMENTAL MECHANICS.

Two of the expressions located at the lower righthand corner of the figure are incorrect. Expres- sion $M_{x}+\frac{\partial Q_{x}}{\partial x} d x$ should be $Q_{x}+\frac{\partial Q_{x}}{\partial x} d x$, and expression $Q_{x}+\frac{\partial M_{x}}{\partial x} d x$ is correctly $M_{x}+\frac{\partial M_{x}}{\partial x} d x$.

The Editors 